\title{
Effects of Walls and Floors in Indoor Localization Using Tracking Algorithm
}

\author{
Farhat M. A. Zargoun \\ Computer and Systems Engineering \\ department \\ Faculty of Engineering, Zagazig \\ University, Egypt
}

\author{
Ibrahim M. Henawy \\ Computer Science department \\ Faculty of computer and information, \\ Zagazig University, Egypt
}

\author{
Nesreen I. Ziedan \\ Computer and Systems Engineering \\ department \\ Faculty of Engineering, Zagazig \\ University, Egypt
}

\begin{abstract}
The advancement in wireless and mobile networks has led to an increase in location based services (LBS). LBS can be applied in many applications, such as vehicle systems, security systems, and patient tracking systems.

The Global Navigation Satellite Systems (GNSS) have become very popular due to their ability to provide highly accurate positions, especially in outdoor environments. However, GNSS signals become very weak when they go through natural or manmade structure, like in urban canyons or indoor environments. This hinders the applicability of GNSS-based localization techniques in such challenging environments.

Many indoor localization techniques are based on the received signal strength (RSS). An RSS is proportional to the distance to an access point (AP), where it is stronger in power when it is closer to an AP, given that the received signal is not obstructed by walls or floors. This paper aims at studying the effect of walls and floors on the RSS, and estimating the distribution of the RSS due to such obstructions. Moreover, a tracking algorithm based on a multi-walls and floors propagation model is applied to increase the positioning accuracy.
\end{abstract}

Keywords-Indoor localization; Tracking algorithm; Effects of wall and floor on RSS; Effects of obstruction; Multi wall and floor propagation model

\section{INTRODUCTION}

The received signal strength (RSS) from a transmitter to a receiver is proportional to the distance between them. Therefore, the RSS has been an important measure in wireless positioning techniques. Three transmitters, or access points (AP), are required to estimate the $3-\mathrm{D}$ position of a receiver.

The study provided in this paper takes into account walls and floors as obstructions that attenuate the RSS. The losses in the RSS depend on materials that makeup walls and floors, signals can go through walls or floors, but these signals suffer from attenuation that depends on the type and thickness of the walls and floors.RSS in indoor locations is difficult to predict due to the attenuation effects. However, signal losses can be measured using a multi walls and floors propagation model.

The remainder of this paper is organized as follows. First, the path loss and attenuation due to walls and floors are introduced. Following that, an experiment that illustrates the distribution of RSS is presented. A method is then presented to predict the location using RSS. The effect of multi-walls and multi-floors on the RSS is investigated next, and used in an algorithm to estimate the location in an indoor environment.
Finally, experiments are performed to investigate the validity of the presented algorithm.

\section{RELATED WORK}

In [4] K-Nearest Neighbor (KNN) algorithm is used to predict the user's location in a single floor environment. KNN works as a matching technique that compares a measured RSS with RSS stored in a radio map, and concludes the position as the one that has stored RSS closest to the measured RSS. The matching can be done by using metric techniques such as the Euclidean distance [4]. The estimated location accuracy is affected by the size of reference points and k-value.

The authors in [5] designed an indoor localization system by using Bayesian graphical model. This model depends on the set of conditional independence relationships. The first model of Bayesian network is used by [6], which was a simple model to estimate the user's location for a single floor. Sampling techniques, which are needed for the Bayesian model, are calculated by using collected data. One of the most important sample techniques is based on the Markov Chain Monte Carlo (MCMC) [6].

In indoor environments, walls and floors attenuate the RSS and hinder the position estimation.

According to [7][9], the signal strength is affected by the types of concrete walls. Some types of walls have a high attenuation factor, so that each floor can reduce the received signal strength between 10 and $35 \mathrm{dBm}$. This factor plays a main role in estimating a user's location in a multi-floor environment. There are some propagation mechanisms that need to be determined in order to investigate the characteristics of propagation in a multi-floor environment, such as multipath diffraction, reflection, and scattering. The diffraction or scattering due to a window frame or an edge of a building wall can also affect the FAF. Moreover, the attenuation of the RSS depends on the size of the window frame in each floor.

\section{PATH LOSS AND ATTENUATION}

A path loss is a measure of the average signal attenuation. It can be expressed as:

$$
P_{L}=P_{L 0}+10 n \log (d)
$$

Where

$P_{L}$ - Attenuation due to multi walls and floors. 


$$
P_{L_{0}} \text { - Path loss at distance of } 1 \text { meter. }
$$

$n$ - Power-low relationship between distance and received power.

$d$ - Distance between a transmitter and a receiver.

In order to take into account the attenuation due to walls and floors, two additional terms are added to (1). The new equation is called multi walls and floors propagation equation [1]. The relationship between penetration loss and the number/type of floors and walls can be expressed by:

$$
P L_{m w f}=P L_{0}+10 n \log (d)+\sum_{i=1}^{I} \sum_{k=1}^{K_{w i}} P L_{w i k}+\sum_{j=1}^{J} \sum_{k=1}^{K_{f}} P L_{f j k}
$$

$P_{L_{m w f}}$ - Attenuation due to multi walls and floors.

$P_{L_{0}}$ - Path loss at distance of 1 meter.

$n$ - Power-low relationship between distance and received power.

$d$ - Distance between transmitter and receiver.

$P_{L_{w i k}}$ - Attenuation due to wall type I and Kth traversed walls.

$P_{L_{f j k}}$ - Attenuation due to floor type $\mathrm{j}$ and Kth traversed floors.

i - Number of walls.

j - Number of floors.

$K_{w i}$ - Number of traversed walls.

$K_{f j}$ - Number of traversed floors.

\section{DistRIBUTION OF RSS}

To design an indoor localization system, one needs to study the properties of the RSS. RSS is difficult to predict due to factors such as multipath effects and obstructions, Figures 2(a) and 2(b) show the measured RSS to 2 access points (APs). The experiment was performed in the first floor in the Training Center (TC), in the faculty of electronic engineering, BaniWalid, Libya. The walls are made of wood that has iron bars inside. The RSS were measured at fixed locations for 50 minutes, with 10 seconds interval, during work hours. The figures show that each experiment is different from each the other. This is because of the difference in the distance between the sender and the receiver, and because of obstructions.

Two experiments are conducted to test the difference between normal log of signal propagation.

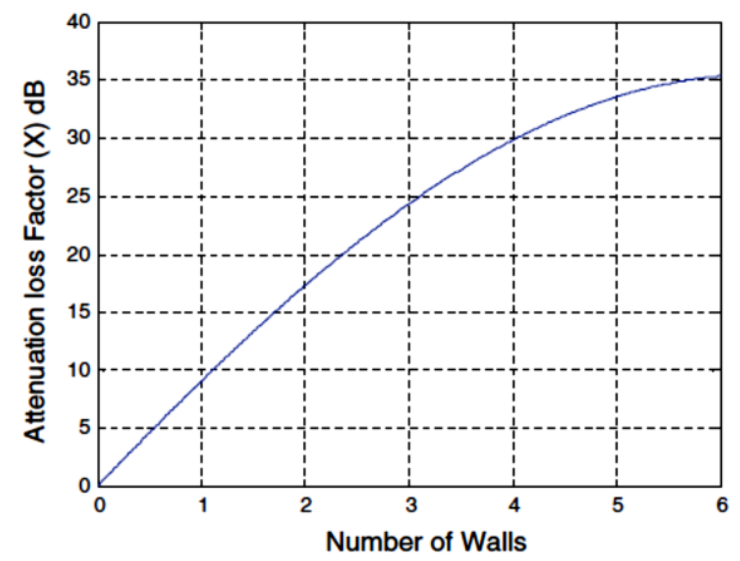

Fig. 1. Best fit curve of the average curves of the attenuation loss factor

One of them when there is a line of sight (LOS) between the transmitter and the receiver, and another one show how the obstruction can affect the signal [13].

The experiment setup is two access points (reference nodes) and one laptop computer (user node), Xirrus WiFi inspector software is installed in user node to capture the signal strength. The RSS is measured from two different distances from each AP, in the presence of obstructions such as walls and floors. Another experiment is conducted to measure the RSS without obstruction. Figures 3(a), 3(b) show the results.

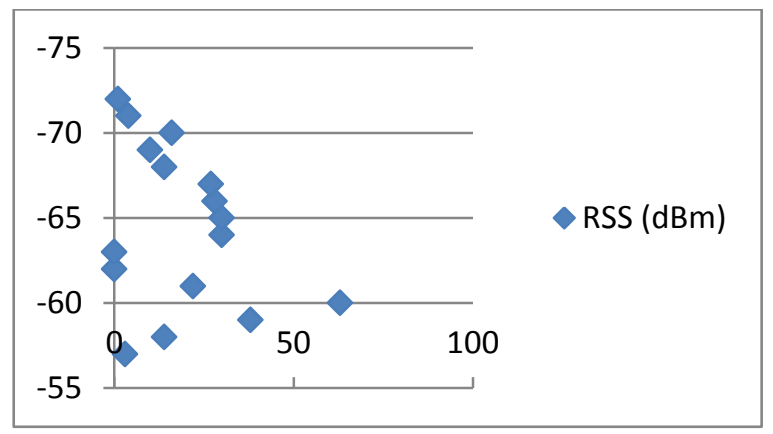

Fig. 2. (a) AP 1, LOS

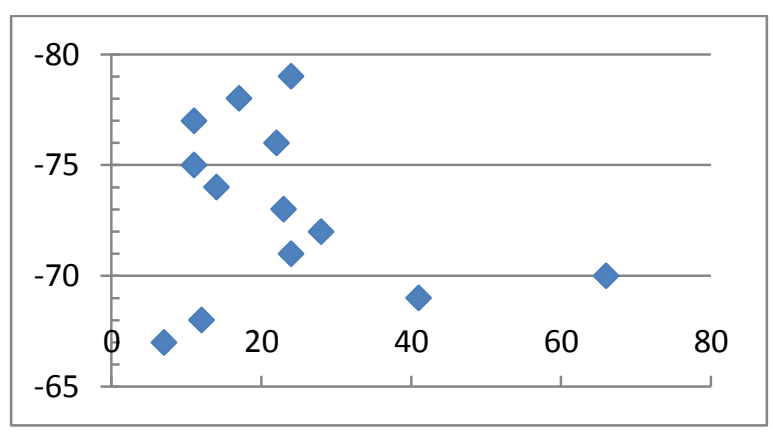

Fig. 2. (b) AP 1, NLOS 


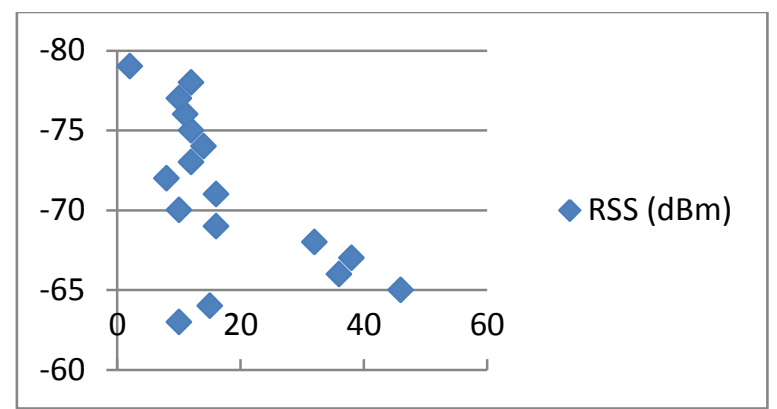

Fig. 3. (a) AP 2, LOS

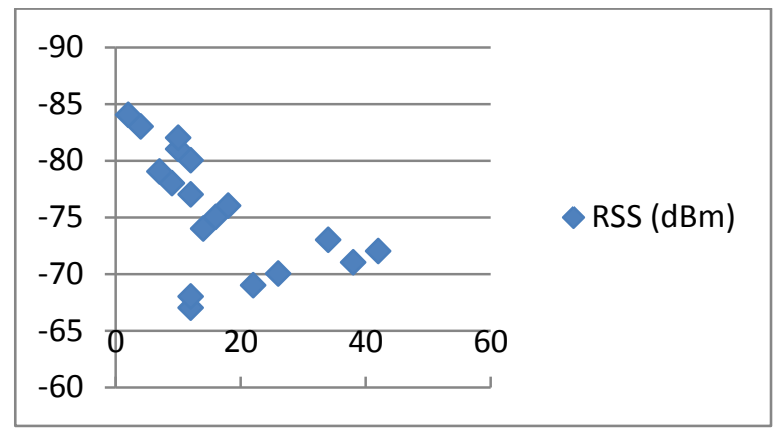

Fig. 3. (b) AP 2, NLOS Histogram of RSS for two access points

\section{USING RSS TO PREDICT THE LOCATION}

RSS can be used as a reference to predict the indoor location. A test is conducted to show the RSS variability due to distance and obstruction. Figure (3) shows the variation of the RSS measurements recorded from four APs, where two of them are located in the first floor, and the others are located in the second floor [13]. The measurements are recorded by a person walking through a track in the first floor at the Training Center Building. The signal received at any given location is stronger when the location is close to the AP and weaker when it is far away. This shows the feasibility of using RSS as a location Fingerprint. Figure (5) shows the uniqueness of the RSS tuples. Each RSS tuples at each location are different. This indicates that RSS fingerprints are a reasonable choice for inferring indoor locations. Figure (5) also shows that there is a small variation in the RSS when the distance between two training points is short.

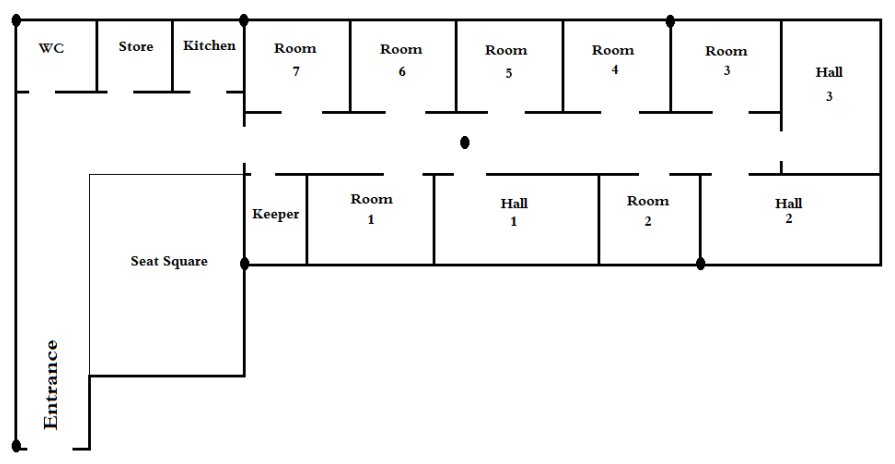

Fig. 4. Training Center Building

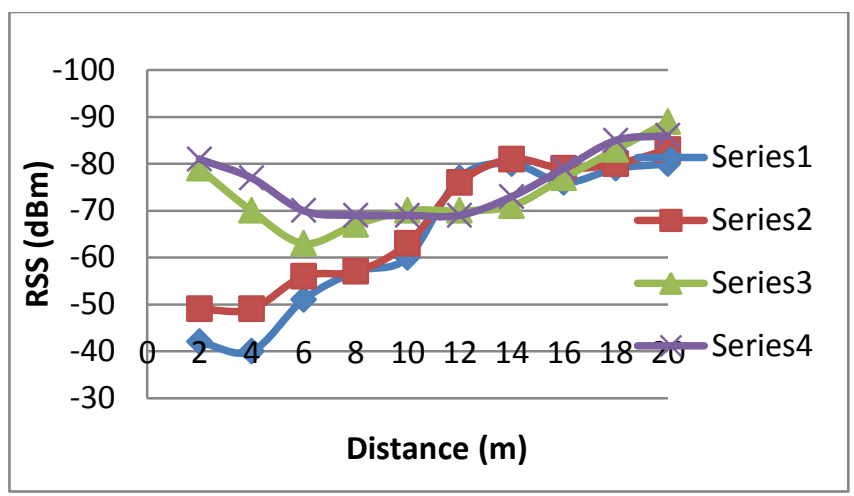

Fig. 5. RSS while walking

Table (1) shows the RSS statistics from the four APs at two different floor

TABLE I. RSS STATISTICS

\begin{tabular}{||l||l||l||l||l||}
\hline Statistics & $\begin{array}{l}\text { AP 1 } \\
\text { 1st } \\
\text { floor }\end{array}$ & $\begin{array}{l}\text { AP 2 } \\
\text { 1st } \\
\text { floor }\end{array}$ & $\begin{array}{l}\text { AP 3 } \\
\text { 2nd } \\
\text { floor }\end{array}$ & $\begin{array}{l}\text { AP 4 } \\
\text { 2nd } \\
\text { floor }\end{array}$ \\
\hline \hline $\begin{array}{l}\text { Mean } \\
\text { Measured }\end{array}$ & -64.2 & -67.3 & -73.9 & -75.8 \\
\hline $\begin{array}{l}\text { Mean } \\
\text { Calculated }\end{array}$ & -61.8 & -63.4 & -68.1 & -71.2 \\
\hline $\begin{array}{l}\text { Standard } \\
\text { Deviation }\end{array}$ & 1.2 & 1.95 & 2.9 & 2.3 \\
\hline Min. & -40 & -49 & -63 & -69 \\
\hline Max. & -80 & -83 & -89 & -86 \\
\hline
\end{tabular}

\section{Multi Wall EFFECT}

According to [1], the reduction in RSS depends on type of the wall. Table (2) shows the attenuation in the RSS due to different types of walls.

TABLE II. DiFFERENT TYPES OF WALLS ATtENUATION

\begin{tabular}{|l|l|l|}
\hline Wall type and thickness & First wall & Second wall \\
\hline Concrete $10 \mathrm{~cm}$ & $16 \mathrm{db}$ & $14 \mathrm{db}$ \\
\hline Concrete $20 \mathrm{~cm}$ & $29 \mathrm{db}$ & $24 \mathrm{db}$ \\
\hline
\end{tabular}

An experiment is performed in another building, to show the effect of concrete walls. The walls have $20 \mathrm{~cm}$ thickness. 3 offices are used, where each office has one AP. The signal strength is measured at the middle of each office, where the measurements are done over 5 minutes with 3 seconds intervals, Figure(7) shows the result of this experiments. 


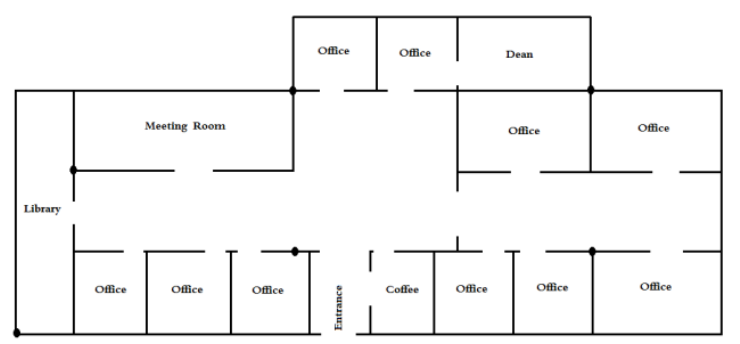

Fig. 6. Administration Building

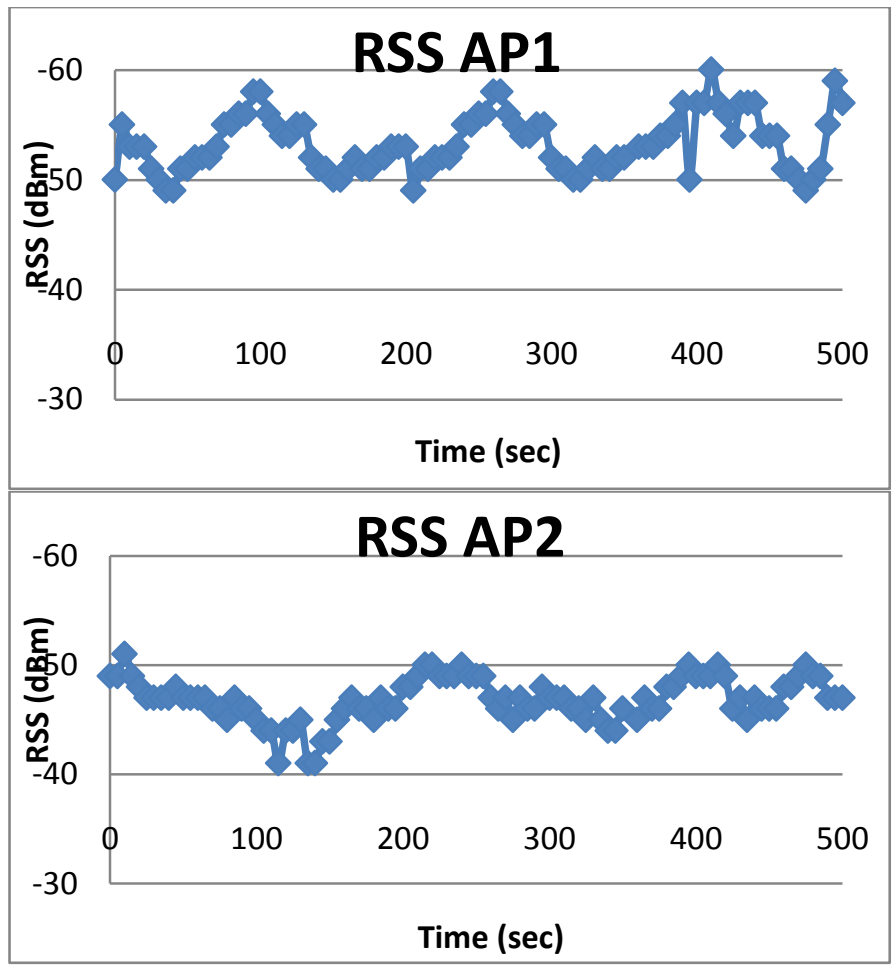

Fig. 7. Effects of walls

\section{MULTI FLOOR EFFECT}

According to [8], table (3) shows the attenuation in RSS caused by floors. Another experiment is conducted to check the attenuation caused by floors. The floor is concrete with $22 \mathrm{~cm}$ thickness. The experiment is done in the second floor. 4 APs are placed on the ground, in the first and third floors, to get the same distance between each AP and a Laptop located in the second floor. RSS measurements are collected at a fixed location, over 5 minutes, with 3 seconds intervals. Figure (8) shows the effect of concrete floors.

TABLE III. FlOORS ATtENUATION

\begin{tabular}{|l|l|}
\hline Floor 2,1 & $15.4 \mathrm{db}$ \\
\hline Floor 2,3 & $18.7 \mathrm{db}$ \\
\hline
\end{tabular}

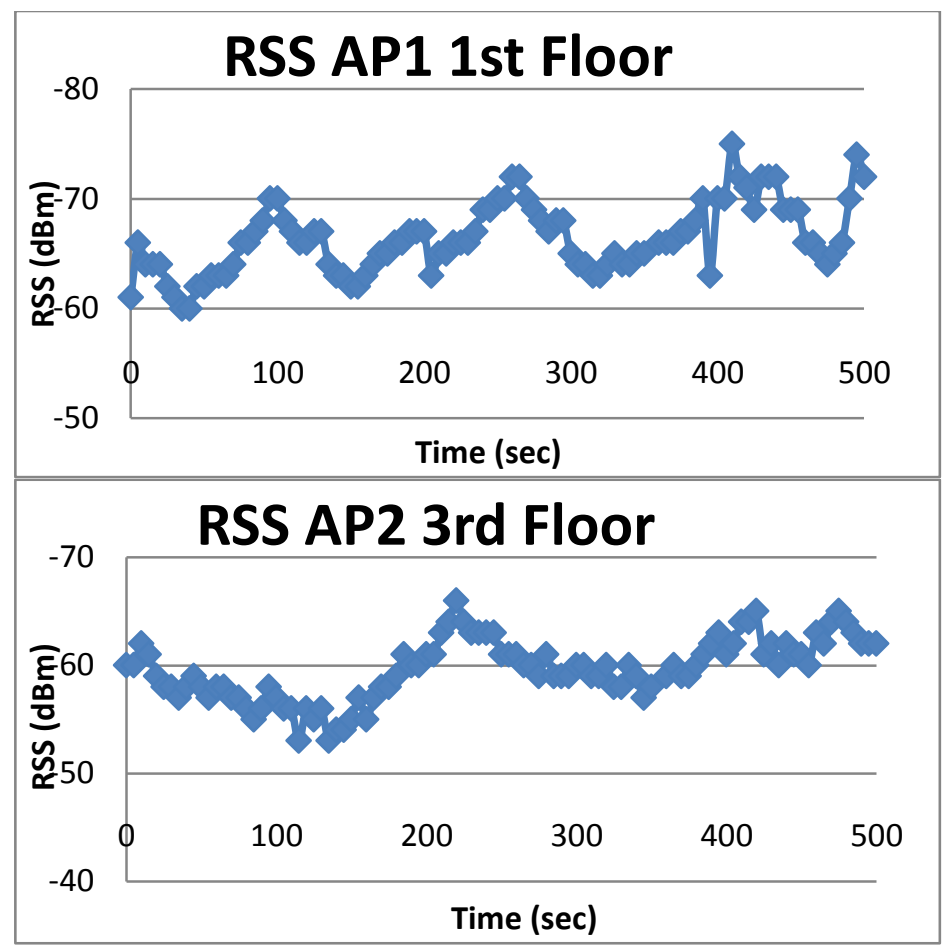

Fig. 8. Effects of concrete floors

\section{SYSTEM MODEL}

Ranging error, which occurs due to obstructions that attenuate the signal, is a major difficulty in indoor localization algorithms. A technique is proposed to overcome this error. The technique works in two modes, which are range-based and range-free modes. The technique first estimates the locality of a user node, which depends on the number of reference nodes in range.

In the second step the locality information is used as input to the second part of the algorithm. The algorithm then collects data about the number and type of obstructions (Table 2 and 3), and finally uses multi wall and floor propagation model in (5) to estimate the location of user node.

The proposed technique requires the storage of the building layout and the attenuation loss of the obstructions (walls and floors). This technique can be implemented in either a centralized manner or in a distributed manner.

In the first step, the algorithm checks whether the user node is in the coverage area of the reference node or not. The check is passed when the distance between the user node and the reference node is less than the maximum radius of the coverage area.

If a user node, in special cases, receives signals from only 2 reference nodes, it can find its locality based on the geometry theory as shown in Figure (9) [8]. 


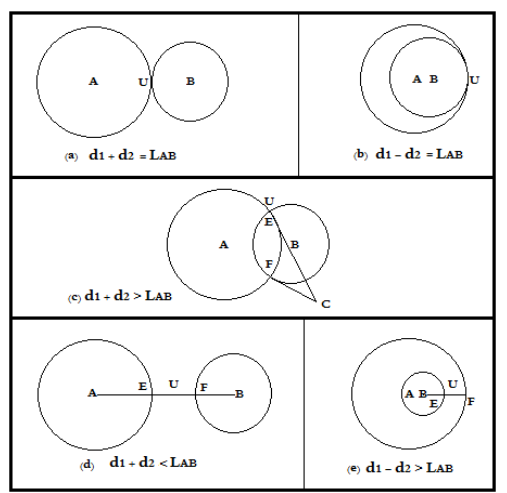

Fig. 9. five cases using two circle to determine the location of the unknown node

When the two circles are externally tangent or internally tangent as shown in Figures 9(a) and (b), the estimated intersection point of the unknown node is taken. If the two circles intersect, there are two intersection points as shown in Figure 9(c). In this case, a revised RSS between the third beacon node and the unknown node as the reference is used to compare $\left|d_{3}-C E\right|$ with $\left|d_{3}-C F\right|$ and select the smaller one as the estimated unknown node. Here, RSS from the third beacon node is utilized as a reference to identify the proper position between two intersection points.

In in Figures 9(d) and (e), there may be no intersection point because of measurement error. In these cases, we take the middle point of and as the unknown node E F.

\section{ALGORITHM}

- Estimate the locality of the user node from the intersection of the strongest reference nodes signals.

$$
\begin{aligned}
& \text { For all user node I; } \\
& \text { Loc_indicator }=0 ; \\
& \text { For each reference node } j \\
& \qquad \begin{array}{l}
\text { If }\left(x_{j}-x_{i}\right)+\left(y_{j}-y_{i}\right)<=R_{j}^{2} \\
\text { Loc_indicator }++1 ;
\end{array}
\end{aligned}
$$

- Collect data about the number and type of walls and floors between the estimated user node and each reference node.

- Use the multi wall and floor propagation model to correct the location of user node.

\section{EXPERIMENTAL SETUP}

A series of experiments are conducted in the Training center building and the Administration building. Some of the experiments are done in 2-D by keeping the reference and user nodes at the same height, while other experiments are done in 3 -D. The first set of experiments is done in the training center building, which has 7 reference nodes, and one user node that captures RSS via Xirrus WiFi inspector software. The location of the user node is estimated using the proposed first part of the algorithm.

The second set of experiments is done in the administration building, which has 12 reference nodes. 6 reference nodes are on the ground of the first floor, and 6 reference nodes are on the ground of the third floor. The error in the estimated location is calculated as the distance between the actual and estimated locations as expressed in (3).

$$
\text { Error }=\sqrt{\left(X_{e s t}-X\right)^{2}+\left(Y_{e s t}-Y\right)^{2}}
$$

\section{EXPERIMENT RESULTS}

In each experiment, 10 locations are chosen in each building to calculate the error before and after adding the effect of walls and floors. Experimental results show that the position estimation error is less than 2 meters for most locations.

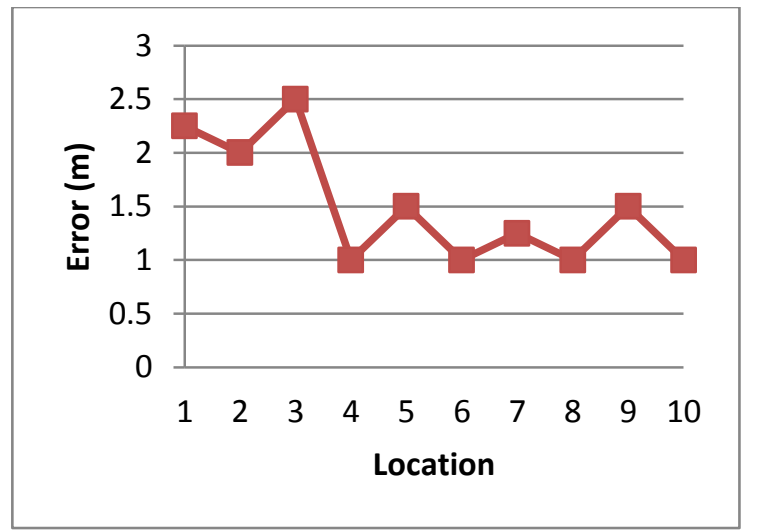

Fig. 10. Location estimation error for various locations in training center building

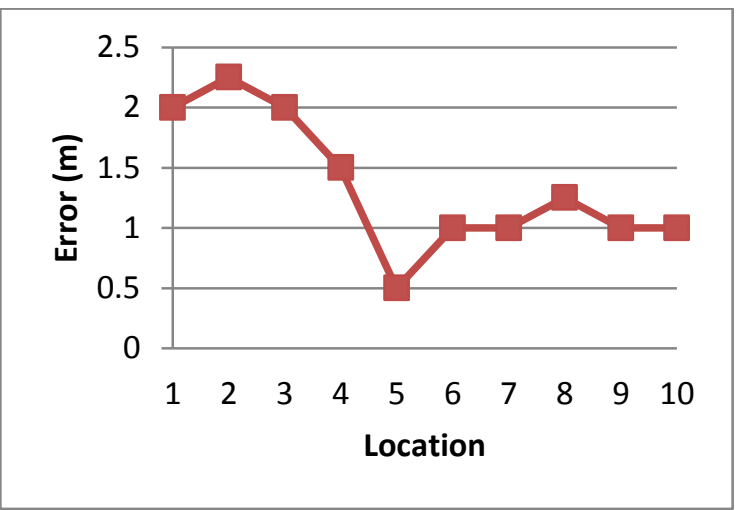

Fig. 11. Location estimation error for various locations in administration building

\section{CONCLUSION}

Currently there is no indoor localization system that can provide an accurate position estimation for all environments. This paper provides an experiment that illustrates the distribution of RSS is presented. A method is then presented to predict the location using RSS. Also, the paper provides a study on the effects of walls and floors on the RSS, and investigates a localization technique that utilizes the finding of the effects of obstructions on the RSS. The investigated technique uses multi walls and floors model in the estimation. The system model showed the major difficulty in indoor localization algorithm, especially the difficulty of location estimation using the storage of building layout, the algorithm has been used to estimate location using three or more 
reference nodes, and in special cases, by using two reference nodes. Experimental results show that the position estimation error is less than 2 meters for most locations. It is suitable for indoor environments with multiple floors and multiple walls.

\section{REFERNECE}

[1] Lott, M. and Forkel, I. "A Multi wall and floor for indoor radio propagation", Vehicular Technology Conference, Volume 1, 464-468, 2001.

[2] Kaemarungsi, K. and P. Krishnamurthy, "Properties of indoor received signal strength for WLAN location fingerprinting", Proceedings of the 1st Annual International Conference on Mobile and Ubiquitous Systems: Networking and Services (MOBIQUITOUS04), 14-23, 2004.

[3] Komar, C. and C. Ersoy, "Location tracking and location based service using IEEE 802.11 WLAN infrastructure", European Wireless, 24- 27, 2004.

[4] Binghao, L. , James, S. ; Andrew, G. D. and Chris, R. "Indoor positioning techniques based on wireless LAN", First IEEE International Conference on Wireless Broadband and Ultra Wideband Communications, Sydney, Australia. 2006.

[5] Al-Ahmadi, A. S. ; Omer, A. I. ; Kamarudin, M. R. and Rahman, T. A. "multi-floor indoor positioning system using bayesian graphical models", Progress In Electromagnetics Research B, Vol. 25, pp. 241$259,2010$.
[6] Elnahrawy, E. ; Martin, R.W. ; Ju, P. K. and Madigan, D. "Bayesian indoor positioning systems", Infocom. Citeseer, pp. 1217- 1227, 2005.

[7] Komar, C. and Ersoy, C. "Location tracking and location based service using IEEE 802.11 WLAN infrastructure", European Wireless, pp. 2427, 2004.

[8] Xiao Fan and Yoan Shin, "Indoor Localization for Multi-Wall, MultiFloor Environments in Wireless Sensor Networks", AICT 2013.

[9] Hossain, A. K.; Hien, N. V ; Yunye, J. and Seng, S. W. "Indoor Localization Using Multiple Wireless Technologies", in Mobile Adhoc and Sensor Systems, 2007. MASS 2007. IEEE Internatonal Conference, pp. 1-8, 2007.

[10] Baala, O. ; You, Z. and Caminada, A. "The Impact of AP Placement in WLAN-Based Indoor Positioning System", in Networks, 2009. ICN '09. Eighth International Conference, pp. 12-17, 2009.

[11] A. Fink, H. Beikirch, M. Voss, and C. Schroeder. RSSI-based Indoor Positioning using Diversity and Inertial Navigation. In IEEE International Conference on Indoor Positioning and Indoor Navigation (IPIN), pages 1-7, 2010.

[12] Seow, C. and S. Tan, "Localization of omni-directional mobile device in multipath environments", Progress In Electromagnetics Research, Vol. $85,323\{348,2008$

[13] Pandey, S. and P. Agrawal, "A survey on localization techniques for wireless networks", Journal Chinese Institute of Engineers, Vol. 29, No. 7, 1125, 2006. 